\title{
Chronic Inflammatory Mediators Induced Malignant Changes in Tumor Microenvironment of Oral Squamous Cell Carcinoma-New Insight
}

\author{
T.G. Shrihari*
}

\author{
Department of Oral Medicine and Oral Oncology, Krishna Devaraya College of Dental Sciences and \\ Research Center, Bangalore-562157, Karnataka, India
}

\begin{abstract}
Oral cancer is a major cause of mortality and morbidity across the world. Because of extensive use of carcinogenic products such as Tobacco (Smoking or Chewable form), alcohol consumption and some cases due to infectious agents such as HPV (Human papilloma virus) induced oro-pharyngeal carcinoma. These carcinogens induce inflammatory changes in the inflammatory microenvironment of oral cavity. Oral Squamous cell carcinoma is the most common cancer of oral cavity. Chronic inflammatory mediators in tumor microenvironment are adaptive and innate immune cells such as macrophages, neutrophils, T- lymphocytes, mast cells,B-lymphocytes and their secreting factors such as proteases, ROS and cytokines, which in turn activates transcriptional factors (NF-KB,STAT-3) secreted by these immune cells and tumor cells bring about malignant changes. This article briefs about chronic inflammatory mediators in tumor microenvironment of oral Squamous cell carcinoma and their role in tumor progression.
\end{abstract}

Keywords: Cytokines, Growth factors, NF-KB, STAT-3, TGF- $\beta$, PAMP, DAMP, Tumor associated macrophages, Tumor associated neutrophils, T lymphocytes, B lymphocytes, HIF-1 $\alpha$, AP-1, Tregs.

\section{INTRODUCTION}

Oral cancer is a sixth most common cancer in the world. Worldwide annual incidence of 300,000 new cases of oral cancer, of which $62 \%$ of cases are from developing countries and annual death rate of 1,45,328 cases worldwide. More than $74 \%$ of oral cancers are developed from potentially malignant lesions and conditions. According to globocan 2012, oral cancer accounts $16,88,50$ new cases and 97,408 death rate annually in Asia. Major causes of oral cancer are tobacco, alcohol consumption and infectious agents such as HPV (Human Papilloma Virus). Most of the cases diagnosed later in stage III and IV are known to be identified with increased mortality and morbidity [14].

Highest cases of oral cancer reported in South east asian countries such as Sri Lanka and India. In Indian subcontinent incidence and mortality rate of oral cancer is $1 / 3$ rd of the global burden. Chronic inflammation accounts 20 percent of overall cancer burden. Oral Squamous cell carcinoma is the most common cancer of oral cavity, mostly preceded by oral potentially malignant lesions and conditions [1-5].

Inflammation is the body's defensive mechanism against chemical, thermal and infectious agents. If it is

*Address correspondence to this author at the Department of Oral Medicine and Oral Oncology, Krishna Devaraya College of Dental Sciences and Research Center, Bangalore-562157, Karnataka, India; Tel: 91-9844386188; E-mail: drshrihariomr@gmail.com dysregulated, aggravates chronically, can cause offence by various inflammatory mediators to the host. Chronic inflammatory mediators present in tumor microenvironment of oral squamous cell carcinoma are innate immune cells such as neutrophils, macrophages, mast cells, dendritic cells, natural killer cells, and adaptive immune cells such as $T$ lymphocytes, B lymphocytes generates cytokines, chemokine's, growth factors and proteolytic enzymes [6] (Figure 1).

\section{CHRONIC INFLAMMATORY CELLS AND THEIR FACTORS IN PROGRESSION OF CANCER}

Inflammation mainly involves innate immune cells, triggered by viral structures and foreign microbes known as specialized recognition pattern PAMP (Pathogen associated molecular pattern) or cell death and tissue injury releases cellular constituents known as DAMP (Damage associated molecular pattern), recognized by PRR (Pattern recognition receptors) which belongs to TLR (Toll like receptor) trans membrane receptor family. TLR transmits the intracellular signal through adaptor protein (My88), results in further activation of innate and adaptive immune cells, amplifying inflammatory response. Activation of innate immunity results in secretion of chemokines and cytokines, recruit $\mathrm{T}$ cells and MHC class 1 and class 11 up regulation.

Chemokine's are chemotactic cytokines that involve in positioning and migratory patterns of immune cells to the site of inflammation. Receptors of chemokine's are 


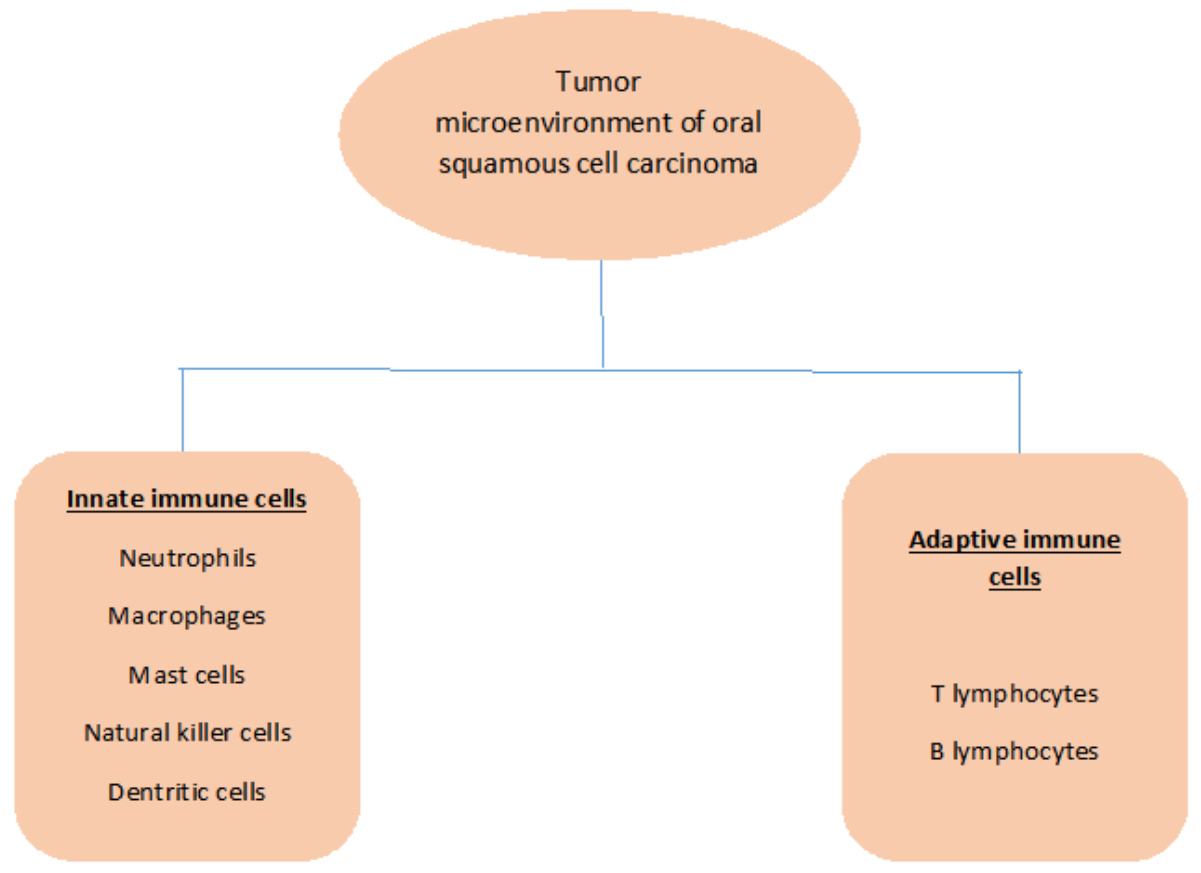

Figure 1: Show Immune cells present in tumor micro environment of oral sequamous cell carcinoma.

expressed on leucocytes produced by stromal and tumor cells facilitates tumor progression. Neutrophil recruitment is mediated by CXCL1, CXCL2, CXCL3, CXCL5, CXCL6, CXCL7, CXCL8, recruitment of macrophages are by CCL2, CXCL12-CXCR4, CCL4, CCL5, MCP-1 chemokine's. Lymphocyte recruitment by chemokine's CXCL12-CXCR4, CXCL9, CXCL10, CXCL11, CCR7-CCL21, CXCL19, CCL21.

Adaptive immunity upregulates $\mathrm{T}$ and $\mathrm{B}$ lymphocytes. Thus, Th1 cells (Type1 helper $T$ cells) secrete IFN- $\Upsilon$ by activation of macrophages in a cellto- cell contact manner. Eosinophil's get activated by Th2 cells through cytokine release and antibodies secreted by $B$ cells, which in turn, activate the complement cascade, phagocytes, NK cells (Natural killer cells) and mast cells [7, 8].

Chronic inflammatory cells in tumor microenvironment can bring about malignant changes, in which the predominant cells are tumor associated macrophages, diverse and exhibit high level of plasticity. Tumor associated macrophages are divided functionally and phenotypically into two subtypes classically activated (M1) and alternatively activated (M2). M1 macrophages have immune stimulatory activity by Th1 cytokines such as $1 \mathrm{~L}-2$, IFN- $\Upsilon$, TNF- $\alpha$, whereas M2 macrophages have an immunosuppressive action and tumor progression by Th2 cytokines such as IL-4, IL-5, IL-6, and IL-13. In tumor microenvironment, M1 macrophages (TAM1) express and secrete IL-1, IL-6, TNF- $\alpha$, IL-23 and iNOS, which contribute to tumor initiation and promotion.

Tumor associated macrophages 2(M2 phenotype) accumulate under hypoxic cores of tumor microenvironment and produce IL-10, TGF-Beta and arginase-1. Activation of HIF-1 $\alpha$ transcription factor under hypoxic condition stimulates the expression of genes which encode proteins promoting inflammatory reactions such as VEGF, iNOS, COX2, IL-8 (CXCL8), thereby induces angiogenesis and immunosuppression. Tumor associated macrophages 2 (M2 phenotype) promote angiogenesis by secreting (GM-CSF, VEGF, EGF and angiopoietin 1 and 2), invasion by secreting proteases (such as MMP-2, 9 ,cathepsin B, D) and also chronic inflammation by secreting PGE-2 produced by COX-2 and reactive oxygen species (ROS), reactive nitrogen species (RNS). These ROS and RNS free radicals may cause damage to DNA and cell mutation through aberrant expression of oncogenes and suppression of tumor suppressor genes. Activation of NF-kB by cytokines IL1 , TNF- $\alpha$, STAT3 by IL-6, EGF, are the critical transcriptional factors and HIF-1 $\alpha$, AP-1 by TNF- $\alpha$, IL$1 \beta$, induce malignant changes involved in cellular proliferation by Cyclin D, C-myc, cell cycle regulatory proteins resistant to apoptosis (Cell survival) by (BCL$\mathrm{xL}, \mathrm{BCL}-2)$, Immunosuppression/inflammation by (MHC-1,MHC-11, and IL-10,TGF- $\beta$ pro-inflammatory cytokines) angiogenesis by (IL-8, VEGF, COX-2), invasion and metastasis by (TGF- $\beta$, MMP-2,9, upA) 


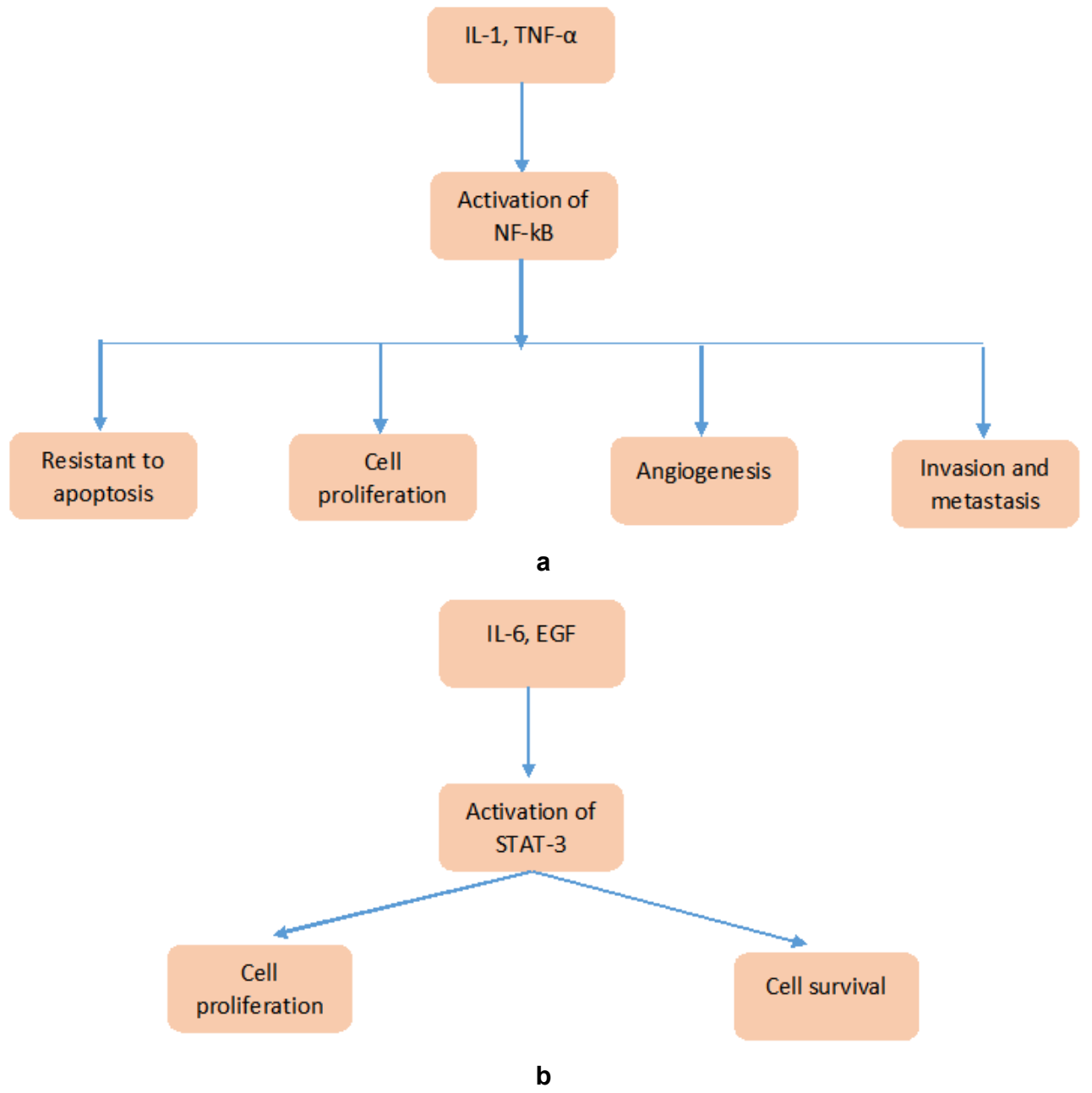

Figure 2: Show the transcription factors activated inflammatory mediators involved in the progression of oral squamous cell carcinoma.

(Figure 2a and 2b). IL-10 and TGF- $\beta$, the main immunomodulatory cytokines of M2 macrophages, produce CCL22 that attracts Treg (regulatory T cells), which maintain immunosuppressive microenvironment [9-11].

Oncogenic stress induces IL-8 release from the epithelial cell that attracts neutrophils to the inflammatory site. Inflammatory cytokine TGF- $\beta$ regulates polarization of $\mathrm{N} 1$ to protumoral N2. Tumor associated neutrophils co-relate with angiogenesis, tumor growth and metastasis.

Tumor associated neutrophils secrete ROS (Reactive oxygen species), RNS (Reactive nitrogen species) lead to oxidative stress, causing DNA damage, genomic instability and matrix degrading proteases such as MMPs promotes tumor growth. Granulocytes are produced from bone marrow, but in inflammatory process, their precursors myelocytes and premyelocytes exit to peripheral circulation. In premetastatic niche, granulocytes secrete prokinectin 2 to attract tumor cells and protease MMP9, induce VEGF secretion from neighbor cells to facilitate the arrival of tumor cells. Growth factor PDGF promote tumor cell proliferation produced by granulocytes [8].

Mast cells are players of innate and adaptive immunity originate from hematopoietic system, modulate major immune response. Differentiated and mature in vascularized tissues, it is an important cell component in a tumor microenvironment brought to the tumor site by stem cell factor necessary for maturation, migration and survival of mast cells and other chemo attractants such as FGF-2, VEGF. These release angiogenic mediators, growth factors and proteases that support tumor development. Proteases released by mast cell such as tryptase, activate matrix metalloproteases, contribute to extracellular matrix degradation, vascular tube formation, promote angiogenesis and metastasis. They secrete cytokines, which in turn stimulate NF- kB that antagonizes the 
activity of p53 by activating the enzyme AID(Activated induced deaminase), favor tumor growth by angiogenesis, resistance to apoptosis, increase cell proliferation, genomic instability and metastasis [12-15].

Tumor stroma maintains the immunosuppressive environment by factors including hypoxia, accumulation of extracellular adenosine, lactate, IL-6, M-CSF, VEGF, which, all together inhibit dendritic cell antigen presenting activity by TGF- $\beta$ responsible for immunosuppression. Dendritic cells express high level of IDO (Indole amine 2,3 -dioxygenase), recruits T cells, TGF- $\beta$ induces differentiation into Treg cells, maintain immunosuppressive environment that favor tumor progression [16-18].

CD8 T cells secrete IFN- $\Upsilon$ helps M1 macrophage polarization, triggers cytotoxic or phagocytic activities. Th1 cytokines are secreted by CD4 T cells helps in cytotoxic and phagocytic activities. IL-4, IL-5, IL-6 and IL-13 pro inflammatory cytokines are secreted by Th2 cells, which inhibit Th1 response, Th17 cells promote inflammatory conditions induce by IL-23 proinflammatory cytokine. Treg cells inhibit innate and adaptive immune cells, Th1, Th2 or Th17 cells. Therefore, CD8 or CD4 Th1 cells favor antitumor activities, while Treg cells, Th2 and Th17 cells favor protumoral or immunosuppressive conditions $[8,14]$.

Treg (regulatory $\mathrm{T}$ cells) is of two types natural Tregs mature in thymus and inducible Tregs is mediated by TGF- $\beta$ enriched inflammatory environment. Treg cells exert immune-modulatory role by TGF- $\beta$, IL-10 cytokines and cell to cell contact via CTLA4. Cytotoxic $T$ cell and dendritic cell maturation arrest by TGF- $\beta$ and IL-10 immunosuppressive cytokines. IL-10 is an inhibitor of NF-KB, STAT / JAK signaling pathways by expression of IL-3, IL-2, TNF- $\alpha$ and GM- CSF cytokines [16].

Bregs (regulatory $B$ cells) induced tolerance by producing IL-10 and/or TGF-Beta, down regulate inflammatory reactions, and inhibit pathogenic $T$ cell immune response. Among different markers IL-10 acts as a $B$ cell biomarker used for identification of Bregs, potent deactivator limits inflammatory response. B cells produce cytokine such as lymphotoxin and immunoglobulins such as IgA, complement mediated chronic inflammation and activates mast cells and myeloid cells, which required for tumor progression in tumor microenvironment of oral squamous cell carcinoma [8, 19-21].
TGF- $\beta$ produced by B-cell, mediated suppression of cellular immune responses. TGF- Beta is a pleiotropic cytokine produced by cancer and stromal cells including fibroblasts and immune cells, acts as an antiinflammatory, immunosuppressive and tumor promotive activity through activating SMAD transcription factor involved in invasion and metastasis by inducing epithelial to mesenchymal transition (EMT). IL-10 and TNF- $\alpha$ required by $\mathrm{B}-$ cell to mediate $\mathrm{TH} 2$ activation and inhibition of cytotoxic activity of $\mathrm{CD} 8^{+} \mathrm{T}$ cells induce onco-promotion. B-cells secrete antibodies and immune complexes that would stimulate recruitment of myeloid and mast cells at tumor sites promote tumor growth. Bcell secreting IL-10 are B regs, $B$ cell subset $B$ regs secrete antibody and cytokines, which induce the immune complex production and tumor progression by stimulating cell signaling molecules signal. Bregs by inhibiting the cytotoxic activity of Th1/CD8 $T$ cells, could promote cancer growth mediated by IL-10 and TGF- $\beta$ production [21].

Inflammatory cells in tumor microenvironment of oral squamous cell carcinoma involved in tumor initiation, promotion and progression. Inflammatory cells includes innate immune cells such as neutrophils, macrophages, mast cells, NK cells and dendritic cells, adaptive immune cells such as $\mathrm{T}$ and $\mathrm{B}$ lymphocytes present in tumor microenvironment of oral squamous cell carcinoma secrete immune mediators such as cytokines, growth factors, proteolytic enzymes, which activates transcription factors such as NF-kB, STAT-3, HIF-1 $\alpha$ and AP-1 bring about angiogenesis, cell proliferation, cell survival, immunosuppression, invasion and metastasis.

\section{CONCLUSION}

Thorough understanding of each role of innate and adaptive immune cells and their mediators, interaction with cancer cells and stroma tumor microenvironment of oral squamous cell carcinoma microenvironment and their signaling pathway in tumor progression for better prognostic and future therapeutic strategy.

\section{ABBREVIATIONS}

HGF = Hepatic growth factor

VEGF = Vascular endothelial growth factor

MMP-9 = Matrix mettaloproteinases-9

COX2 = Cyclo-oxygenase2 


\begin{tabular}{|c|c|c|c|}
\hline INOS & $=$ Inducible nitric oxide synthase & GM-CSF & $\begin{aligned} &= \text { Granulocyte Macrophage- Colony } \\
& \text { stimulating factor }\end{aligned}$ \\
\hline ROS & $=$ Reactive oxygen species & & \\
\hline PDGF & $=$ Platelet derived growth factor & G-CSF & $=$ Granulocyte colony stimulating factor \\
\hline EGF & $=$ Epidermal growth factor & STAT3 & $\begin{aligned}= & \text { Signal transducer and activator of } \\
& \text { transcription } 3\end{aligned}$ \\
\hline FGF & $=$ Fibroblast growth factor & bFGF & $=$ basic fibroblast growth factor \\
\hline TNF-Alfa & $=$ Tumor necrosis factor-Alfa & MMPS & $=$ Matrix metallo proteinases \\
\hline IFN-Beta & $=$ Interferon Beta & HIF-1 Alfa & $=$ Hypoxia- Inducible factor Alfa \\
\hline IL-10 & $=$ Interleukin 10 & T reg cell & $=\mathrm{T}$ regulatory cell \\
\hline TGF-Beta & $=$ Transforming growth factor- Beta & Th1 & $=\mathrm{T}$ helper 1 \\
\hline CCL17 & $=\mathrm{CC}$ Chemokine ligand 17 & Th2 & $=\mathrm{T}$ helper 2 \\
\hline CCL18 & $=\mathrm{CC}$ Chemokine ligand 18 & TAM & $=$ Tumor associated macrophages \\
\hline CCL22 & $=\mathrm{CC}$ chemokine ligand 22 & $A C$ & GEMENTS \\
\hline
\end{tabular}$$
\text { PGE2 = Prostaglandin E2 }
$$$$
\text { IDO= = Indole amine 2,3 -dioxygenase }
$$$$
\text { UPA } \quad=\text { Urokinase plasminogen activator }
$$$$
\text { UPAR = Urokinase plasminogen activator }
$$$$
\text { receptor }
$$$$
\mathrm{IL}-2=\text { Interleukin } 2
$$$$
\text { IL-4 = Interleukin } 4
$$$$
\text { IL-6 = Interleukin }-6
$$$$
\text { IFN-Gamma = Interferon Gamma }
$$$$
\text { COX2 = Cyclo-oxygenase } 2
$$$$
\text { NF-KB = Nuclear factor KB }
$$$$
\text { MCP-1 = Macrophage/Monocyte chemoattractant }
$$$$
\text { protein-1 }
$$$$
\text { M-CSF = Macrophage colony stimulating factor }
$$$$
\text { IL-17 = Interleukin } 17
$$$$
\text { CD4+ Th17 = CD4+ Thelper lymphocyte17 }
$$$$
\text { MDSC = Myeloid derived suppressor cells }
$$$$
\text { SR-A = The class } \mathrm{A} \text { macrophage scavenger }
$$
receptor msr1

I would like to thank Dr. Soundarya for her help in proof reading the article.

\section{CONFLICT OF INTEREST STATEMENT}

Authors have declared no conflicts of interest.

\section{FUNDING SOURCES}

This research did not receive any specific grant from funding agencies in the public, commercial, or notfor-profit sectors.

\section{REFERENCES}

[1] Mc Cormack VA, Boffetta P. Today's lifestyles, tomorrow's cancers; Trends in lifestyle risk factors for cancer in low- and middle - income countries. Ann Oncol 2011; 22: 2349-57. https://doi.org/10.1093/annonc/mdq763

[2] Tabishur R, Ghulam SH. Oral cancer awareness in cancer capital of india: An insight. International Journal of Current Research 2018; 10(2): 650510-645054.

[3] Anand $P$, Kunnumakkara $A B$, Sundaram $C$, Harikumar $K B$, Tharakan ST, Lai OS, et al. cancer is a preventable disease that requires major lifestyle changes. Pharm Res 2008; 25: 2097-116.

https://doi.org/10.1007/s11095-008-9661-9

[4] Warnakulasuriya S. Global epidemiology of oral and oropharyngeal cancer. Oral Oncol 2009; 45: 309-16. https://doi.org/10.1016/j.oraloncology.2008.06.002

[5] Jemal A, Siegel R, Xu J, Ward E. Cancer statistics, 2010. CA Cancer J Clin 2010; 60: 277-300. https://doi.org/10.3322/caac.20073

[6] Elango JK, Sundaram KR, Gangadharan P, Subhas P, Peter $S$, Pulayath $C$, et al. Factors affecting oral cancer awareness in a high - risk population in India. Asian Pac J Cancer Prev 2009; 10: 627-30. 
[7] Vishakaha G, Anoop K, Komal S, Gagandeep K. Chronic inflammation and carcinogenesis-Emerging role of chronic inflammatory periodontal disease. Cancer Research Frontiers 2016; 2(2): 200-225. https://doi.org/10.17980/2016.200

[8] Sahingur SE, Yeudall WA. Chemokine function in periodontal disease and oral cavity cancer. Front Immunol 2015; 6: 214229.

https://doi.org/10.3389/fimmu.2015.00214

[9] Shabnam S, Michael K. Immunity, Inflammation, and cancer: an eternal fight between good and evil. The Journal of Clin Invest 2015; 125(9): 3347-3355. https://doi.org/10.1172/JCl80007

[10] Sheppard K, Kinross KM, Solomon B, Pearson RB, Phillips WA. Targeting $\mathrm{PI} 3$ kinase/AKT/mTOR signaling in cancer. Critical Reviews in Oncogenesis 2012; 17: 69-95. https://doi.org/10.1615/CritRevOncog.v17.i1.60

[11] Pollard JW. Trophic macrophages in development and disease. Nature Reviews Immunology 2009; 9: 259-270. https://doi.org/10.1038/nri2528

[12] Hao NB, Lu MH, Fan YH, Cao YL, Zhang ZR, Yang SM. Macrophages in tumor microenvironments and the progression of tumors. Clinical \& Developmental Immunology 2012; 24: 450-461. https://doi.org/10.1155/2012/948098

[13] Thiago TM, Ivan CM, Olivier $\mathrm{H}$. The role of mast cells in cancers. F100 Prime Rep 2015; 7: 9.

[14] Shrihari TG, Vijeev V, Soundarya K, Deepa Reddy, Manjunath V. Devaraju D. Anti-inflammatory dietary supplements in the chemoprevention of cancer. Cancer Research Frontiers 2016; 2(3): 380-395. https://doi.org/10.17980/2016.380
[15] Grivennikov SI, Greten FR, Karin M. Immunity, Inflammation, and Cancer. Cell 2010; 6: 883-899. https://doi.org/10.1016/j.cell.2010.01.025

[16] Shrihari TG, Vasudevan V, Manjunath V, Devaraju D. Potential Co-relation between chronic periodontitis and cancer- An emerging concept. Gulf Journal of Oncology 2016; 1(20): 20-24.

[17] Korniluk A, Koper O, Kemona H, Dymicka- Piekarska. From inflammation to cancer. Irish Journal of Medical Science 2016; 10: 45-52.

[18] Burkholder B, Huang RY, Burgess R, Luo S, Jones VS, Zhang W et al. Tumor -induced perturbation of cytokines and immune cell networks. Biochimica ET Biophysica ActaReviews on Cancer 2014; 2: 182-201. https://doi.org/10.1016/j.bbcan.2014.01.004

[19] Chimal-Ramirez GK, Espinoza-Sanchez NA, FuentesPanana ES. Protumor Activities of the immune response: Insights in the mechanisms of Immunological shift, oncotraining, and oncopromotion. Journal of Oncology 2013; 7: 1-16. https://doi.org/10.1155/2013/835956

[20] Gabrilovich DI, Ostrand-Rosenberg S, Bronte V. Coordinated regulation of myeloid cells by tumours. Nature Reviews Immunology 2012; 12: 253-268. https://doi.org/10.1038/nri3175

[21] Colotta F, Allavena P, Sica A, Garlanda C, Mantovani A. Cancer-related inflammation,the seventh hallmark of cancer: links to genetic instability. Carcinogenesis 2009; 30(7): 107381. https://doi.org/10.1093/carcin/bgp127

[22] Mantovani A. Macrophages, Neutrophils and cancer: A double edged sword. New Journal of Science 2014; 8: 1-14. https://doi.org/10.1155/2014/271940 